\title{
Soil Quality Mapping Studies Using Nematodes as Bioindicators
}

\author{
Lakshmy Kamala Sthanu $^{1 *}$, Jaya Divakaran Sarasamma ${ }^{2}$, Radhakrishnan Thanu Iyer ${ }^{3}$, \\ Mohandas Chellappan ${ }^{4}$
}

${ }^{1}$ Centre for Environment and Development, Thozhuvancode, Vattiyoorkavu P.O., Thiruvananthapuram, India; ${ }^{2}$ Department of Environmental Sciences, University of Kerala, Kariavattom Campus, Thiruvananthapuram, India; ${ }^{3}$ Division of Geo Informatics, Indian Institute of Information Technology and Management, Kerala, Techno Park Campus, Thiruvananthapuram, India; ${ }^{4}$ Division of Crop Protection, Central Tuber Crops Research Institute, Sreekaryam, Thiruvananthapuram, India.

Email: "hariluxmi@gmail.com, jayvijayds@gmail.com,rkequinox@gmail.com,drcmohandas@gmail.com

Received October $5^{\text {th }}, 2013$; revised November $5^{\text {th }}, 2013$; accepted November $12^{\text {th }}, 2013$

Copyright (C) 2013 Lakshmy Kamala Sthanu et al. This is an open access article distributed under the Creative Commons Attribution License, which permits unrestricted use, distribution, and reproduction in any medium, provided the original work is properly cited.

\begin{abstract}
Soil quality is one of the most important factors in sustaining the global biosphere and developing sustainable agricultural practices. Land use and management practices greatly impact the direction and degree of soil quality changes in time and space. Understanding the effects of land use and management practices on soil quality and its indicators has been identified as one of the most important goals for modern soil science. Soil quality mapping study represents a method for assessing and mapping soil quality changes in time and space in small units. For the present study, changes in the physical, chemical parameters and nematode density of the soils in the rural and urban areas of Thiruvananthapuram district, Kerala, were determined. The soil samples were collected from seven different categories of contaminated soils namely coastal area, sewage disposal area, industrial area, road-side area, agricultural area, market area and gasoline station area, and also from two control stations in rural and urban areas. The soil physico-chemical parameters and nematode density were determined. Geostatistics combined with GIS was applied to analyze the spatial variability of soil physico-chemical characteristics and nematode density. This soil quality mapping study provides a basis for identifying tension zones and serves as a triggering mechanism for implementation of soil contamination mitigating strategies.
\end{abstract}

Keywords: Soil Quality; Mapping Studies; Nematode Density; Spatial Variability; Contaminated Soils

\section{Introduction}

Industrialization and urbanization have a strong impact on the environment, and its biotic and abiotic components. Many toxins added to the soils by different anthropogenic activities can build up to concentrations that become serious threats to plant and animal health (Alloway, 1996) [1]. The presence or absence of indicator species or indicator community reflects environmental conditions. Bio monitors provide a direct measurement of a biological effect rather than inferring values using soil extractions (Tarazona et al., 2005) [2]. The study of regional variations and the anthropogenic contamination by metals of soils is very important for environmental planning and monitoring in urban areas. Mapping allows for more efficient approach to remediation and monitoring of soil contaminants (For-

"Corresponding author. tin and Dale, 2005) [3]. Geographical Information System (GIS) is used for the mapping of the contaminants in the study area soils. Based on the GIS analysis, the highest risk sites are identified in which majority of the remediation and monitoring should take place. The major objective of the present study was to monitor the soil quality of selected rural and urban areas in Thiruvananthapuram District, Kerala, using nematodes as bioindicators, and also aimed to provide base line information on the soil health status of rural and urban areas of the capital district of Kerala.

\section{Materials and Methods}

\subsection{Study Area}

The rural and urban areas in Thiruvananthapuram District, Kerala were selected for the present study (http: www. censusindia.net/2001 census results). Thiruvananthapuram 
District is located at $8^{\circ} 26^{\prime} 25^{\prime \prime} \mathrm{N}$ latitude and $76^{\circ} 55^{\prime} 25^{\prime \prime} \mathrm{E}$ longitude. Thiruvananthapuram, the southernmost District in the State of Kerala is the study area. Its southern most extremity, Kaliaikkavila, is only 54 kms away from Kanyakumari (Cape Comarin), the land's end of India. The district stretches $78 \mathrm{kms}$ along the shores of the Arabian Sea on the west, Kollam district lies on the north with Thirunelveli and Kanyakumari districts of Tamil Nadu on the east and the south respectively. The district can be divided into three geographical regions; highlands, midlands and lowlands. The large forest reserves favorably affect the climate and induce rain. Cold weather is experienced in the mountain ranges whereas lower down, the weather is bracing and in the plains, it is generally hot. Though the mean maximum temperature is only around $900 \mathrm{~F}$, it is oppressive in the moisture-laden atmosphere of the plains. Humidity is high and rises to about 90 percent during the south-west monsoon. The average rainfall is around $150 \mathrm{~cm}$ per annum. It is significant that the district gets rainfall both from the south-west and the north-east monsoons. According to the final figures of 2011 census (Census of India, 2011), the population of the district is 3,307,284. The Taluks in Thiruvananthapuram District are Neyyattinkara, Thiruvananthapuram, Nedumangad and Chirayinkeezhu. The district has three major rivers, several freshwater lakes and more than 300 ponds. The eastern region is forested, northern regions are mostly under rubber cultivation and the remaining areas have mixed dry land crops of coconut, plantain, tapioca, etc. Built up areas and rice fields complete the land use. Based on the land use pattern, Thiruvananthapuram District has been classified into different categories namely, coastal areas, sewage disposal areas, industrial areas, road-side areas, agricultural areas, market areas, gasoline station areas, benign environment, etc. These areas will be receiving different types of wastes based on the source of contamination. The contaminated sites due to different anthropogenic activities were identified in both rural and urban areas of Thiruvananthapuram District after conducting a reconnaissance survey. Sixteen sampling stations in the rural and urban areas of the Thiruvananthapuram District were selected. These include seven different categories of contaminated soils selected in the rural and urban areas namely coastal area, sewage disposal area, industrial area, roadside area, agricultural area, market area and gasoline station area. Control soil samples were collected from comparatively benign environments in both rural and urban areas which were selected as control stations (Table 1).

\subsection{Soil Collection}

Soil samples $(0-15 \mathrm{~cm}$ depth) from the sampling stations were collected during the pre-monsoon, monsoon and post-monsoon seasons of the study period (April 2009 to January 2010). Composite samples were taken by mixing the samples collected from five different sites of each station. Soil sampling and analysis of soil physico-chemical characteristics were carried out according to the standard procedures (Gupta, 1999) [4]. The concentration of the heavy metals viz. lead, chromium, manganese, copper and zinc in the acid digested soil samples were determined (Saxena, 1994) [5] using an Atomic Absorption Spectrophotometer (Model, GBC 932 AA). Karl Pearson's Product Moment Correlation is used for finding significant correlation between nematode densities with all other soil quality parameters in the three different seasons (SPSS v - 16 for WINDOWS).

The Geographical coordinates of the sampling stations were collected using Hand-held GPS (Garmin eTrex Vista $\mathrm{HCx}$ ). The Coordinate values were entered into a spreadsheet with properly assigning IDs for each location. Another spreadsheet was prepared with physico-chemical characteristics and nematode density. In this spreadsheet, the same IDs were entered against respective stations. Both spreadsheets were added in GIS software (Arc GIS version 10) as tables. The table with Geographic coordinate values were plotted as point layer using "Display $\mathrm{XY}$ " method and subsequently exported as a shape file with WGS84 as reference ellipsoid. The physico-chemical characteristics and nematode density table was joined with the attribute table of the point layer using ID as the foreign key using JOIN method in GIS software (Korte, 2001) [6]. The resultant layer comprised the geometry of stations as points and all the results as associated attribute table (Figure 1). The soil physico-chemical characteristics were analyzed and mapping of significantly correlated soil physico-chemical characteristics with nematode density was plotted.

\section{Results and Discussion}

\subsection{Mapping of Physico-Chemical Characteristics and Nematode Density in Soil}

The results of physical characteristics of the soils in the study stations are given in Tables 2(a) and (b) respectively and the soil chemical characteristics analyzed are given in Tables 3(a) and (b) respectively. The results of heavy metal $(\mathrm{Pb}, \mathrm{Zn}, \mathrm{Mn}, \mathrm{Cu}, \mathrm{Cr})$ content in soil are given in Tables 4(a) and (b) respectively. Correlation studies of soil physico-chemical characteristics with nematode density were done.

\subsection{Nematode Sampling, Extraction and Density Calculation}

One kg soil samples from 0 - $15 \mathrm{~cm}$ depth were collected. For soil nematode identification studies, $100 \mathrm{cc}$ (approx. $150 \mathrm{gms}$ ) of soil samples were washed and the soil solutions were passed through 60 mesh $(250 \mu)$ and 350 mesh 
Table 1. Portrait of sampling stations in the study area.

\begin{tabular}{|c|c|c|c|c|c|}
\hline \multicolumn{3}{|c|}{ Rural Stations } & \multicolumn{3}{|c|}{ Urban Stations } \\
\hline \multirow{2}{*}{ Name } & \multicolumn{2}{|c|}{ Location } & \multirow{2}{*}{ Name } & \multicolumn{2}{|c|}{ Location } \\
\hline & Latitude & Longitude & & Latitude & Longitude \\
\hline $\begin{array}{l}\text { Vilappilssala } \\
\text { (Station 2) }\end{array}$ & $8^{\circ} 30^{\prime} 18^{\prime \prime}$ & $76^{\circ} 53^{\prime} 45^{\prime \prime}$ & $\begin{array}{l}\text { Valiathura } \\
\text { (Station 10) }\end{array}$ & $8^{\circ} 33^{\prime} 51^{\prime \prime}$ & $76^{\circ} 53^{\prime} 25^{\prime \prime}$ \\
\hline $\begin{array}{l}\text { Kochuveli } \\
\text { (Station 3) }\end{array}$ & $8^{\circ} 30^{\prime} 18^{\prime \prime}$ & $76^{\circ} 53^{\prime} 45^{\prime \prime}$ & $\begin{array}{c}\text { Peroorkkada } \\
\text { (Station 11) }\end{array}$ & $8^{\circ} 31^{\prime} 35^{\prime \prime}$ & $76^{\circ} 58^{\prime} 03^{\prime \prime}$ \\
\hline $\begin{array}{l}\text { Mangalapuram } \\
\text { (Station 4) }\end{array}$ & $8^{\circ} 30^{\prime} 18^{\prime \prime}$ & $76^{\circ} 53^{\prime} 45^{\prime \prime}$ & $\begin{array}{l}\text { Thampanoor } \\
\text { (Station 12) }\end{array}$ & $8^{\circ} 29^{\prime} 15^{\prime \prime}$ & $76^{\circ} 57^{\prime} 12^{\prime \prime}$ \\
\hline $\begin{array}{c}\text { Palode } \\
(\text { Station 5) }\end{array}$ & $8^{\circ} 28^{\prime} 53^{\prime \prime}$ & $76^{\circ} 57^{\prime} 08^{\prime \prime}$ & $\begin{array}{l}\text { Sreekaryam } \\
\text { (Station 13) }\end{array}$ & $8^{\circ} 30^{\prime} 18^{\prime \prime}$ & $76^{\circ} 53^{\prime} 45^{\prime \prime}$ \\
\hline $\begin{array}{l}\text { Balaramapuram } \\
\quad(\text { Station 6) }\end{array}$ & $8^{\circ} 27^{\prime} 06^{\prime \prime}$ & $76^{\circ} 57^{\prime} 21^{\prime \prime}$ & $\begin{array}{c}\text { Chalai } \\
\text { (Station 14) }\end{array}$ & $8^{\circ} 28^{\prime} 52^{\prime \prime}$ & $76^{\circ} 57^{\prime} 08^{\prime \prime}$ \\
\hline $\begin{array}{l}\text { Pallichal } \\
\text { (Station 7) }\end{array}$ & $8^{\circ} 27^{\prime} 06^{\prime \prime}$ & $76^{\circ} 57^{\prime} 31^{\prime \prime}$ & $\begin{array}{l}\text { Karamana } \\
\text { (Station 15) }\end{array}$ & $8^{\circ} 28^{\prime} 56^{\prime \prime}$ & $76^{\circ} 57^{\prime} 36^{\prime \prime}$ \\
\hline $\begin{array}{c}\text { Anappara } \\
\text { (Control Station 8) }\end{array}$ & $8^{\circ} 28^{\prime} 53^{\prime \prime}$ & $76^{\circ} 57^{\prime} 08^{\prime \prime}$ & $\begin{array}{c}\text { Ambalathara } \\
\text { (Control Station 16) }\end{array}$ & $8^{\circ} 27^{\prime} 06^{\prime \prime}$ & $76^{\circ} 57^{\prime} 21^{\prime \prime}$ \\
\hline
\end{tabular}

S1 - S8: Rural Sampling Stations; S9 - S16: Urban Sampling Stations.

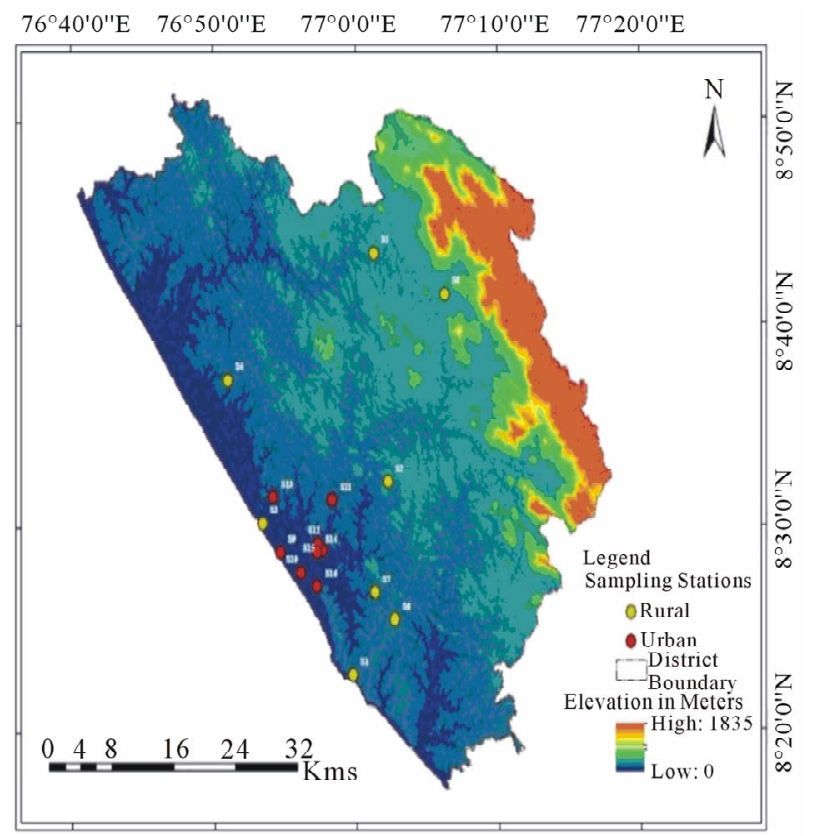

Figure 1. Base map showing the sampling stations in the rural and urban areasof.

(45 $\mu$ ) sieves (ASTM Endecott Sieves) respectively. The residues from 350 mesh sieve was washed and collected into a beaker and allowed to settle for one hour. Then decanted the supernatant and poured the rest on a wet tissue paper placed on metal gauze in a Petri dish containing water. After two days, collected the nematode containing solution, allowed to settle for decanting and added equal quantity of boiled $4 \%$ formalin to kill and fix it. $10 \mathrm{ml}$ of this solution was placed in a nematode Perspex counting dish (Cobb, 1917) [7] and were observed under a Stereomicroscope (Model, Leica LED3000 NVI) and their abundance was calculated (Southwood and Henderson, 2000) [8] during the three seasons as shown in Figures 2(a)-(c). The soil sample solution was made up to $100 \mathrm{ml}$ and stirred well. Pipetted $10 \mathrm{ml}$ of this soil solution from three different depths of this $100 \mathrm{ml}$ soil sample solution kept in a beaker and placed in Doncaster's circular mounting tray (Goodey, 1963) [9]. Nematode density is expressed in Number per $100 \mathrm{ml}$ soil.

Electrical conductivity, water holding capacity, moisture content, organic matter, total nitrogen content, phosphorus content, potassium and copper contents showed positive correlation with nematode density in soil. $\mathrm{pH}$, temperature, pore space, bulk density, chloride, lead, zinc, manganese and chromium contents showed negative correlation with nematode density in soil samples collected from both rural and urban area as shown in Tables 5(a) and (b) respectively. Of the different physicochemical characteristics studied, water holding capacity, moisture content, organic matter content, $\mathrm{pH}$, zinc and manganese contents only showed significant level of correlation with nematode density. Mapping of significantly correlated soil physico-chemical characteristics with nematode density was plotted. The significance level of correlation coefficient is given in Table 6.

\subsection{Mapping of Significantly Positively Correlated Physico-Chemical Characteristics and Nematode Density in Soil}

Water holding capacity of soils in the selected stations of 
Table 2. (a) Physical characteristics of rural and urban soils; (b) Physical characteristics of rural and urban soils.

(a)

\begin{tabular}{|c|c|c|c|c|c|c|c|c|c|}
\hline \multirow[b]{2}{*}{ St.No. } & \multicolumn{3}{|c|}{ pH } & \multicolumn{3}{|c|}{$\begin{array}{l}\text { Conductivity } \\
\text { (mho/cm) }\end{array}$} & \multicolumn{3}{|c|}{$\begin{array}{c}\text { Temperature } \\
\left({ }^{\circ} \mathrm{C}\right)\end{array}$} \\
\hline & $\begin{array}{c}\text { Pre- } \\
\text { monsoon } \\
\text { season }\end{array}$ & $\begin{array}{c}\text { Monsoon } \\
\text { season }\end{array}$ & $\begin{array}{c}\text { Post- } \\
\text { monsoon } \\
\text { season }\end{array}$ & $\begin{array}{c}\text { Pre- } \\
\text { monsoon } \\
\text { season }\end{array}$ & $\begin{array}{c}\text { Monsoon } \\
\text { season }\end{array}$ & $\begin{array}{c}\text { Post- } \\
\text { monsoon } \\
\text { season }\end{array}$ & $\begin{array}{c}\text { Pre- } \\
\text { Monsoon } \\
\text { season }\end{array}$ & $\begin{array}{c}\text { Monsoon } \\
\text { season }\end{array}$ & $\begin{array}{c}\text { Post } \\
\text { monsoon } \\
\text { season }\end{array}$ \\
\hline S1 & $7^{* *}$ & $7.7^{* *}$ & $7.1^{* *}$ & $0.07^{* *}$ & $0.049^{* *}$ & $0.032^{* *}$ & $32.6^{* *}$ & $30^{* *}$ & $31.5^{* *}$ \\
\hline $\mathrm{S} 2$ & $5^{* *}$ & $5.7^{* *}$ & $5^{* *}$ & 1.52 & $0.88^{* *}$ & $0.463^{* *}$ & 30 & 29.7 & 31 \\
\hline $\mathrm{S} 3$ & $5.7^{* *}$ & $6.4^{* *}$ & $6.3^{* *}$ & $0.26^{* *}$ & $0.15^{* *}$ & $0.094^{* *}$ & $32.5^{* *}$ & $30^{* *}$ & $31.5^{* *}$ \\
\hline S4 & $6.1^{* *}$ & $7.1^{* *}$ & $6.5^{* *}$ & $0.26^{* *}$ & $0.082^{* *}$ & $0.057^{* *}$ & $31.6^{* *}$ & $30^{* *}$ & $31.48^{*}$ \\
\hline S5 & $5.3^{*}$ & $6^{*}$ & $5.5^{*}$ & $1.09^{* *}$ & $0.245^{* *}$ & $0.326^{* *}$ & $29.9^{* *}$ & $29^{* *}$ & $31^{* *}$ \\
\hline S6 & $4.7^{* *}$ & $5^{* *}$ & $4.9^{* *}$ & $3.14^{* *}$ & $1.35^{* *}$ & $1.343^{* *}$ & $31.1^{* *}$ & $30^{* *}$ & $31.4^{* *}$ \\
\hline S7 & $6.2^{* *}$ & $7.3^{* *}$ & $6.7^{* *}$ & $0.14^{* *}$ & $0.072^{* *}$ & $0.048^{* *}$ & $33^{* *}$ & $30^{* *}$ & $31.4^{* *}$ \\
\hline S8 & 5.4 & 6.2 & 5.9 & 0.44 & 0.225 & 0.211 & 29.5 & 29.8 & 31 \\
\hline S9 & $8.7^{* *}$ & $8.3^{* *}$ & $7.3^{* *}$ & $0.06^{* *}$ & $0.042^{* *}$ & $0.028^{* *}$ & $32.6^{* *}$ & $30^{* *}$ & $31.5^{* *}$ \\
\hline $\mathrm{S} 10$ & 5.7 & $5.7^{* *}$ & $5.3^{* *}$ & $1.33^{* *}$ & $0.528^{* *}$ & $0.451^{* *}$ & $32^{* *}$ & $30^{* *}$ & $31.3^{* *}$ \\
\hline S11 & 5.7 & 6.3 & 6.2 & $0.27^{* *}$ & $0.174^{* *}$ & $0.099^{* *}$ & $32.2^{* *}$ & $30^{* *}$ & 31.1 \\
\hline $\mathrm{S} 12$ & $6.1^{* *}$ & $7.3^{* *}$ & 6.7 & $0.25^{* *}$ & $0.073^{* *}$ & $0.049^{* *}$ & $31.3^{* *}$ & $30^{* *}$ & $31.4^{* *}$ \\
\hline $\mathrm{S} 13$ & $5.3^{* *}$ & 6.2 & $5.7^{* *}$ & $0.52^{* *}$ & $0.225^{* *}$ & $0.214^{* *}$ & $32^{* *}$ & 29.9 & $31.2^{* *}$ \\
\hline S14 & $4.8^{* *}$ & $5.3^{* *}$ & $4.9^{* *}$ & $1.6^{* *}$ & $1.305^{* *}$ & $0.865^{* *}$ & $31^{*}$ & $30^{* *}$ & $31.3^{* *}$ \\
\hline S15 & $6.9^{* *}$ & $7.5^{* *}$ & $7^{*}$ & $0.07^{* *}$ & $0.064^{* *}$ & $0.04^{* *}$ & $33^{* *}$ & $30^{* *}$ & $31.4^{* *}$ \\
\hline S16 & 5.7 & 6.2 & 6 & 0.33 & 0.197 & 0.118 & 31.1 & 29.8 & 31 \\
\hline
\end{tabular}

(b)

\begin{tabular}{|c|c|c|c|c|c|c|c|c|c|}
\hline \multirow[t]{2}{*}{ St.No. } & \multicolumn{3}{|c|}{ Pore Space (\%) } & \multicolumn{3}{|c|}{ Water holding capacity (\%) } & \multicolumn{3}{|c|}{ Bulk density (g/cc) } \\
\hline & $\begin{array}{l}\text { Pre-monsoon } \\
\text { season }\end{array}$ & $\begin{array}{c}\text { Monsoon } \\
\text { season }\end{array}$ & $\begin{array}{c}\text { Post-monsoon } \\
\text { season }\end{array}$ & $\begin{array}{l}\text { Pre-monsoon } \\
\text { season }\end{array}$ & $\begin{array}{c}\text { Monsoon } \\
\text { season }\end{array}$ & $\begin{array}{c}\text { Post-monsoon } \\
\text { season }\end{array}$ & $\begin{array}{l}\text { Pre-monsoon } \\
\text { season }\end{array}$ & $\begin{array}{c}\text { Monsoon } \\
\text { season }\end{array}$ & $\begin{array}{c}\text { Post-monsoon } \\
\text { season }\end{array}$ \\
\hline S1 & $57.8^{* *}$ & $57.8^{* *}$ & $57.8^{* *}$ & $0.04^{* *}$ & $0.05^{* *}$ & $0.03^{* *}$ & $1.56^{* *}$ & $1.58^{* *}$ & $1.57^{* *}$ \\
\hline $\mathrm{S} 2$ & $30.19^{* *}$ & $30.2^{* *}$ & $30^{*}$ & $1.33^{* *}$ & $1.34^{* *}$ & $1.32^{* *}$ & $0.71^{* *}$ & $0.72^{* *}$ & $0.71^{* *}$ \\
\hline $\mathrm{S} 3$ & $38.32^{* *}$ & $38.2^{* *}$ & $38.1^{* *}$ & 0.15 & $0.17^{* *}$ & $0.15^{* *}$ & $1.14^{* *}$ & $1.14^{* *}$ & 1.2 \\
\hline S4 & $40.12^{* *}$ & $40.13^{* *}$ & $40.1^{* *}$ & $0.15^{* *}$ & $0.15^{* *}$ & $0.14^{* *}$ & $1.23^{* *}$ & $1.24^{* *}$ & $1.23^{* *}$ \\
\hline S5 & $30.28^{* *}$ & $30.3^{* *}$ & $30.2^{* *}$ & $0.79^{* *}$ & $0.75^{* *}$ & $0.74^{* *}$ & $0.5^{* *}$ & $0.9^{* *}$ & $0.9^{* *}$ \\
\hline S6 & $25.43^{* *}$ & $25.4^{* *}$ & $25.3^{* *}$ & $1.84^{* *}$ & $1.87^{* *}$ & $1.86^{* *}$ & $0.2^{* *}$ & $0.3^{* *}$ & $0.3^{* *}$ \\
\hline S7 & $45.25^{* *}$ & $45.3^{* *}$ & $44.9^{* *}$ & 0.13 & $0.13^{* *}$ & $0.11^{* *}$ & $1.26^{* *}$ & $1.25^{* *}$ & $1.25^{* *}$ \\
\hline S8 & 32.45 & 32 & 32 & 0.27 & 0.27 & 0.25 & 1.09 & 1.1 & 1.11 \\
\hline S9 & $58.87^{* *}$ & $58.86^{* *}$ & $58.84^{* *}$ & $0.02^{* *}$ & $0.03^{* *}$ & $0.03^{* *}$ & $1.58^{* *}$ & $1.6^{* *}$ & $1.6^{* *}$ \\
\hline $\mathrm{S} 10$ & $30.19^{* *}$ & $30.2^{* *}$ & $30.1^{* *}$ & $1.23^{* *}$ & $1.25^{* *}$ & $1.23^{* *}$ & $0.8^{* *}$ & $0.8^{* *}$ & $0.78^{* *}$ \\
\hline S11 & $35.45^{* *}$ & $35.5^{* *}$ & $35.2^{* *}$ & $0.18^{* *}$ & 0.2 & 0.2 & $1.11^{* *}$ & 1.12 & $1.15^{* *}$ \\
\hline $\mathrm{S} 12$ & $42.02^{* *}$ & $42.1^{* *}$ & $41.9^{* *}$ & $0.13^{* *}$ & $0.14^{* *}$ & $0.12^{* *}$ & $1.24^{* *}$ & $1.25^{* *}$ & $1.23^{* *}$ \\
\hline $\mathrm{S} 13$ & $31.33^{* *}$ & $31.34^{* *}$ & $31.3^{* *}$ & $0.54^{* *}$ & $0.54^{* *}$ & $0.51^{* *}$ & $0.93^{* *}$ & 1 & $0.97^{* *}$ \\
\hline S14 & $29.26^{* *}$ & $30^{* *}$ & $30^{* *}$ & $1.84^{* *}$ & $1.84^{* *}$ & $1.83^{* *}$ & $0.3^{* *}$ & $0.5^{* *}$ & $0.46^{* *}$ \\
\hline S15 & $48.3^{* *}$ & $48.3^{* *}$ & $48.19^{* *}$ & $0.11^{* *}$ & $0.12^{* *}$ & $0.11^{* *}$ & $1.28^{* *}$ & $1.28^{* *}$ & $1.28^{* *}$ \\
\hline S16 & 35.09 & 35.1 & 34.8 & 0.25 & 0.26 & 0.23 & 1.11 & 1.1 & 1.11 \\
\hline
\end{tabular}

*Significant at $\mathrm{p}<0.05 ;{ }^{* *}$ Significant at $\mathrm{p}<0.01$ level. 
Table 3. (a) Chemical characteristics of rural and urban soils; (b) Chemical characteristics of rural and urban soils.

(a)

\begin{tabular}{|c|c|c|c|c|c|c|c|c|c|}
\hline \multirow[b]{2}{*}{ St.No. } & \multicolumn{3}{|c|}{ Chloride (mg/g) } & \multicolumn{3}{|c|}{ Moisture content (\%) } & \multicolumn{3}{|c|}{ Organic matter (g \%) } \\
\hline & $\begin{array}{l}\text { Pre-monsoon } \\
\text { season }\end{array}$ & $\begin{array}{l}\text { Monsoon } \\
\text { season }\end{array}$ & $\begin{array}{c}\text { Post-monsoon } \\
\text { season }\end{array}$ & $\begin{array}{l}\text { Pre-monsoon } \\
\text { season }\end{array}$ & $\begin{array}{c}\text { Monsoon } \\
\text { season }\end{array}$ & $\begin{array}{l}\text { Post-monsoo I } \\
\text { n season }\end{array}$ & $\begin{array}{c}\text { Pre-monsoon } \\
\text { season }\end{array}$ & $\begin{array}{c}\text { Monsoon } \\
\text { season }\end{array}$ & $\begin{array}{c}\text { Post-monsoon } \\
\text { season }\end{array}$ \\
\hline S1 & $0.34^{* *}$ & $0.338^{* *}$ & $0.339^{* *}$ & $1.74^{* *}$ & $1.81^{* *}$ & $1.76^{* *}$ & $0.517^{* *}$ & $0.591^{* *}$ & $0.569^{* *}$ \\
\hline $\mathrm{S} 2$ & $0.046^{* *}$ & $0.046^{* *}$ & $0.045^{* *}$ & $12.54^{* *}$ & $12.6^{* *}$ & $11.8^{* *}$ & $3.034^{* *}$ & $3.839^{* *}$ & $3.836^{* *}$ \\
\hline $\mathrm{S} 3$ & $0.053^{* *}$ & $0.052^{* *}$ & $0.052^{* *}$ & $5.46^{* *}$ & $5.55^{* *}$ & $5.54^{* *}$ & $1.569^{* *}$ & $1.772^{* *}$ & $1.765^{* *}$ \\
\hline S4 & $0.045^{* *}$ & 0.045 & $0.044^{* *}$ & $4.78^{* *}$ & $4.9^{* *}$ & $4.9^{* *}$ & $1.397^{* *}$ & $1.626^{* *}$ & $1.562^{* *}$ \\
\hline S5 & $0.046^{* *}$ & $0.046^{* *}$ & $0.046^{* *}$ & $9.42^{* *}$ & $9.62^{* *}$ & $9.62^{* *}$ & $2.569^{* *}$ & $3.696^{* *}$ & $3.6^{* *}$ \\
\hline S6 & $0.046^{* *}$ & $0.047^{* *}$ & $0.046^{* *}$ & $15.17^{* *}$ & $15.25^{* *}$ & $15.11^{* *}$ & $5.655^{* *}$ & $5.769^{* *}$ & $4.448^{* *}$ \\
\hline S7 & $0.046^{* *}$ & $0.044^{* *}$ & $0.046^{* *}$ & $2.38^{* *}$ & $2.4^{* *}$ & $2.36^{* *}$ & $1.155^{* *}$ & $1.579^{* *}$ & $1.155^{* *}$ \\
\hline S8 & 0.046 & 0.046 & 0.045 & 8.55 & 8.61 & 8.59 & 1.69 & 2.081 & 2.512 \\
\hline S9 & $0.41^{* *}$ & $0.406^{* *}$ & $0.407^{* *}$ & $0.19^{* *}$ & $0.22^{* *}$ & $0.22^{* *}$ & $0.345^{* *}$ & $0.367^{* *}$ & $0.335^{* *}$ \\
\hline $\mathrm{S} 10$ & $0.047^{* *}$ & $0.047^{* *}$ & $0.046^{* *}$ & $11.85^{* *}$ & $11.9^{* *}$ & $11.5^{* *}$ & $2.672^{* *}$ & $2.838^{* *}$ & $3.836^{* *}$ \\
\hline S11 & 0.046 & 0.046 & 0.045 & $6.79^{* *}$ & $6.82^{* *}$ & $6.76^{* *}$ & $1.569^{* *}$ & $1.94^{* *}$ & $1.867^{* *}$ \\
\hline $\mathrm{S} 12$ & $0.044^{* *}$ & $0.044^{* *}$ & $0.043^{* *}$ & $2.7^{* *}$ & $2.9^{* *}$ & $2.9^{* *}$ & $1.328^{* *}$ & $1.688^{* *}$ & $1.455^{* *}$ \\
\hline $\mathrm{S} 13$ & $0.046^{* *}$ & $0.046^{* *}$ & 0.046 & $8.67^{* *}$ & $8.69^{* *}$ & 8.6 & $2.207^{* *}$ & $2.491^{* *}$ & $2.255^{* *}$ \\
\hline S14 & $0.046^{* *}$ & $0.047^{* *}$ & $0.046^{* *}$ & $13.3^{* *}$ & $14^{* *}$ & $13.6^{* *}$ & $3.5^{* *}$ & $3.881^{* *}$ & $3.87^{* *}$ \\
\hline S15 & $0.046^{* *}$ & $0.045^{* *}$ & $0.046^{* *}$ & $2.35^{* *}$ & $2.36^{* *}$ & $2.29^{* *}$ & $0.69^{* *}$ & $0.909^{* *}$ & $0.905^{* *}$ \\
\hline S16 & 0.046 & 0.046 & $0.046^{* *}$ & 8.06 & 8.1 & 8 & 1.69 & 2.081 & 2.41 \\
\hline
\end{tabular}

(b)

\begin{tabular}{|c|c|c|c|c|c|c|c|c|c|}
\hline \multirow[b]{2}{*}{ St.No. } & \multicolumn{3}{|c|}{$\mathbf{o}$} & \multicolumn{3}{|c|}{ Total Phosphorus (g \%) } & \multicolumn{3}{|c|}{ Total Potassium (g \%) } \\
\hline & $\begin{array}{l}\text { Pre-monsoon } \\
\text { season }\end{array}$ & $\begin{array}{c}\text { Monsoon } \\
\text { season }\end{array}$ & $\begin{array}{c}\text { Post-monsoon } \\
\text { season }\end{array}$ & $\begin{array}{l}\text { Pre-monsoon } \\
\text { season }\end{array}$ & $\begin{array}{c}\text { Monsoon } \\
\text { season }\end{array}$ & $\begin{array}{c}\text { Post-monsoon } \\
\text { season }\end{array}$ & $\begin{array}{l}\text { Pre-monsoon } \\
\text { season }\end{array}$ & $\begin{array}{c}\text { Monsoon } \\
\text { season }\end{array}$ & $\begin{array}{c}\text { Post-monsoon } \\
\text { season }\end{array}$ \\
\hline S1 & $24.5^{* *}$ & $42^{* *}$ & $12.6^{* *}$ & $4.42^{* *}$ & $3.75^{* *}$ & $3.62^{* *}$ & $66.52^{* *}$ & $64.67^{* *}$ & $25.6^{* *}$ \\
\hline $\mathrm{S} 2$ & $63.1^{* *}$ & $105^{* *}$ & $182.7^{* *}$ & $52.46^{* *}$ & $89.82^{* *}$ & $75.31^{* *}$ & $189.73^{* *}$ & $147.41^{* *}$ & $106.1^{* *}$ \\
\hline S3 & $44.8^{* *}$ & $70^{* *}$ & $132.3^{* *}$ & $6.21^{* *}$ & $30.56^{* *}$ & $14.41^{* *}$ & $125.45^{* *}$ & $145.54^{* *}$ & $99.33^{* *}$ \\
\hline S4 & $43.5^{* *}$ & $63^{* *}$ & $119.7^{* *}$ & $5.31^{* *}$ & $27.68^{* *}$ & $13.15^{* *}$ & $106.25^{* *}$ & $143.53^{* *}$ & $90.3^{* *}$ \\
\hline S5 & $59.6^{* *}$ & $91^{* *}$ & $157.5^{* *}$ & $43.57^{* *}$ & $64.11^{* *}$ & $69.21^{* *}$ & $163.39^{* *}$ & $184.6^{* *}$ & $100.7^{* *}$ \\
\hline S6 & $70^{* *}$ & $168^{* *}$ & $252^{* *}$ & $159.82^{* *}$ & $106.43^{* *}$ & $76.36^{* *}$ & $189.73^{* *}$ & $187.32^{* *}$ & $155^{* *}$ \\
\hline S7 & $30.1^{* *}$ & $56^{* *}$ & $107.1^{* *}$ & $4.45^{* *}$ & $7.86^{* *}$ & $5.68^{* *}$ & $83.48^{* *}$ & $128.46^{* *}$ & $60.2^{* *}$ \\
\hline S8 & 49.2 & 77 & 144.9 & 25.76 & 44.64 & 41.65 & 133.04 & 168.86 & 102.88 \\
\hline S9 & $20.3^{* *}$ & $42^{* *}$ & $40.8^{* *}$ & $3.53^{* *}$ & $0.71^{* *}$ & $0.78^{* *}$ & 34.38 & $17.08^{* *}$ & $25.59^{* *}$ \\
\hline S10 & $61^{* *}$ & $98^{* *}$ & $182.7^{* *}$ & $52.46^{* *}$ & $66.07^{* *}$ & $50.2^{* *}$ & $174.55^{* *}$ & $398.44^{* *}$ & $301^{* *}$ \\
\hline S11 & $47^{* *}$ & 70 & $138.6^{* *}$ & $10.67^{* *}$ & $33.21^{* *}$ & $16.41^{* *}$ & 127.23 & $64.51^{* *}$ & $102.34^{* *}$ \\
\hline $\mathrm{S} 12$ & $35.4^{* *}$ & $56^{* *}$ & $107.1^{* *}$ & $5.31^{* *}$ & $14.64^{* *}$ & $7.78^{* *}$ & 85.27 & $43.53^{* *}$ & $84.28^{* *}$ \\
\hline $\mathrm{S} 13$ & $49.6^{* *}$ & $77^{* *}$ & $157.5^{* *}$ & $40.09^{* *}$ & $63.04^{* *}$ & $67.95^{* *}$ & $144.2^{* *}$ & $178.35^{* *}$ & $155^{* *}$ \\
\hline S14 & $64.4^{* *}$ & $154^{* *}$ & $195.3^{* *}$ & $159.82^{* *}$ & $92.14^{* *}$ & $75.52^{* *}$ & $189.73^{* *}$ & $434.82^{* *}$ & $415.5^{* *}$ \\
\hline S15 & $29.6^{* *}$ & $49^{* *}$ & $103.83^{* *}$ & $4.45^{* *}$ & $4.11^{* *}$ & $2.73^{* *}$ & 66.52 & $26.56^{* *}$ & $52.68^{* *}$ \\
\hline S16 & 47.6 & 70 & 144.9 & 12.46 & 41.96 & 27.24 & 130.8 & 85.38 & 106.86 \\
\hline
\end{tabular}

* significant at $\mathrm{p}<0.05 ;{ }^{* *}$ significant at $\mathrm{p}<0.01$ level. 
Table 4. (a) Heavy metal content in rural and urban soils; (b) Heavy metal content in rural and urban soils.

(a)

\begin{tabular}{|c|c|c|c|c|c|c|c|c|c|}
\hline \multirow[b]{2}{*}{ St.No. } & \multicolumn{3}{|c|}{ Pb (mg/kg) } & \multicolumn{3}{|c|}{ Zn (mg/kg) } & \multicolumn{3}{|c|}{ Mn (mg/kg) } \\
\hline & $\begin{array}{l}\text { Pre-monsoon } \\
\text { season }\end{array}$ & $\begin{array}{c}\text { Monsoon } \\
\text { season }\end{array}$ & $\begin{array}{l}\text { Post-monson } \\
\text { season }\end{array}$ & $\begin{array}{l}\text { Pre-monsoon } \\
\text { season }\end{array}$ & $\begin{array}{c}\text { Monsoon } \\
\text { season }\end{array}$ & $\begin{array}{c}\text { Post-monsoon } \\
\text { season }\end{array}$ & $\begin{array}{l}\text { Pre-monsoon } \\
\text { season }\end{array}$ & $\begin{array}{c}\text { Monsoon } \\
\text { season }\end{array}$ & $\begin{array}{l}\text { Post-monsoo } \\
\text { n season }\end{array}$ \\
\hline S1 & BDL & BDL & BDL & 43.2 & 73.1 & 40.3 & 140 & 100.2 & 130.3 \\
\hline S2 & 0.6 & 0.3 & 0.44 & 70.2 & 352.1 & 245.4 & 105.2 & 310.5 & 310.6 \\
\hline S3 & 0.6 & 0.7 & 0.56 & 220.4 & 100.2 & 310.1 & 78.6 & 69.7 & 78.3 \\
\hline S4 & 0.9 & 0.6 & 0.76 & 510 & 220.9 & 535.4 & 401 & 225.6 & 512.6 \\
\hline S5 & BDL & BDL & BDL & 70.4 & 52.3 & 92.3 & 88.6 & 78.4 & 89.3 \\
\hline S6 & 0.6 & 0.6 & 0.4 & 90.1 & 70 & 186.5 & 111.4 & 142.5 & 127.9 \\
\hline S7 & 0.9 & 0.9 & 0.86 & 340.2 & 470.1 & 752 & 540.2 & 398.6 & 567 \\
\hline S8 & $\mathrm{BDL}$ & BDL & BDL & 75.4 & 40.4 & 72 & 70.3 & 63.2 & 70.2 \\
\hline S9 & $\mathrm{BDL}$ & BDL & BDL & 52.3 & 86 & 42 & 157.4 & 109.5 & 510 \\
\hline S10 & 0.8 & 0.3 & 0.4 & 69 & 314 & 252 & 146.4 & 152.3 & 152.1 \\
\hline S11 & 0.6 & 0.7 & 0.61 & 320 & 112.3 & 312.3 & 65.4 & 310.5 & 361.9 \\
\hline $\mathrm{S} 12$ & 0.6 & 0.6 & 0.7 & 360 & 216.9 & 502.9 & 512 & 213.9 & 509.4 \\
\hline S13 & BDL & $\mathrm{BDL}$ & $\mathrm{BDL}$ & 220 & 67.5 & 89.5 & 88.6 & 86.5 & 86.5 \\
\hline S14 & 0.4 & 0.5 & 0.38 & 98.1 & 72.7 & 182.9 & 115 & 152.3 & 138 \\
\hline $\mathrm{S} 15$ & 1 & 0.8 & 0.9 & 360.3 & 508 & 760.4 & 569.8 & 396.5 & 538 \\
\hline S16 & BDL & BDL & BDL & 74.4 & 52.3 & 77.1 & 78.6 & 68.6 & 65.8 \\
\hline
\end{tabular}

(b)

\begin{tabular}{|c|c|c|c|c|c|c|}
\hline \multirow{2}{*}{ St.No. } & \multicolumn{3}{|c|}{$\mathrm{Cu}(\mathrm{mg} / \mathrm{kg})$} & \multicolumn{3}{|c|}{ Cr (mg/kg) } \\
\hline & Pre-monsoon season & Monsoon season & Post-monsoon season & Pre-monsoon season & Monsoon season & Post-monsoon season \\
\hline S1 & 19.2 & 18.7 & 20.5 & 0.2 & $\mathrm{BDL}$ & $\mathrm{BDL}$ \\
\hline S2 & 90.4 & 70.6 & 88.5 & 0.3 & 0.2 & 0.26 \\
\hline $\mathrm{S} 3$ & 79.6 & 71.9 & 77.9 & 0.4 & 0.2 & 0.33 \\
\hline S4 & 78.2 & 74.7 & 75.0 & 0.4 & 0.4 & 0.4 \\
\hline S5 & 79.6 & 76.2 & 77.5 & 0.2 & $\mathrm{BDL}$ & 0.57 \\
\hline S6 & 80.3 & 98.4 & 99.8 & 0.2 & 0.3 & 0.28 \\
\hline S7 & 95.2 & 90.5 & 93.6 & 0.7 & 0.6 & 0.8 \\
\hline S8 & 90.2 & 88.1 & 89.2 & $\mathrm{BDL}$ & $\mathrm{BDL}$ & 0.32 \\
\hline S9 & 20.2 & 18.9 & 20.0 & 0.2 & $\mathrm{BDL}$ & BDL \\
\hline S10 & 92.6 & 89.8 & 90.6 & 0.4 & 0.3 & 0.28 \\
\hline S11 & 86.8 & 85.4 & 85.6 & 0.4 & 0.2 & 0.38 \\
\hline S12 & 82.2 & 81.2 & 82.0 & 0.3 & 0.3 & 0.38 \\
\hline S13 & 87.2 & 83.7 & 86.5 & 0.3 & $\mathrm{BDL}$ & 0.6 \\
\hline S14 & 95.6 & 93.3 & 94.3 & 0.3 & 0.3 & 0.26 \\
\hline S15 & 98.3 & 84.1 & 87.4 & 0.8 & 0.6 & 0.82 \\
\hline $\mathrm{S} 16$ & 88.2 & 83.3 & 85.3 & 0.20 & BDL & 0.31 \\
\hline
\end{tabular}

BDL-Below Detected Level. 


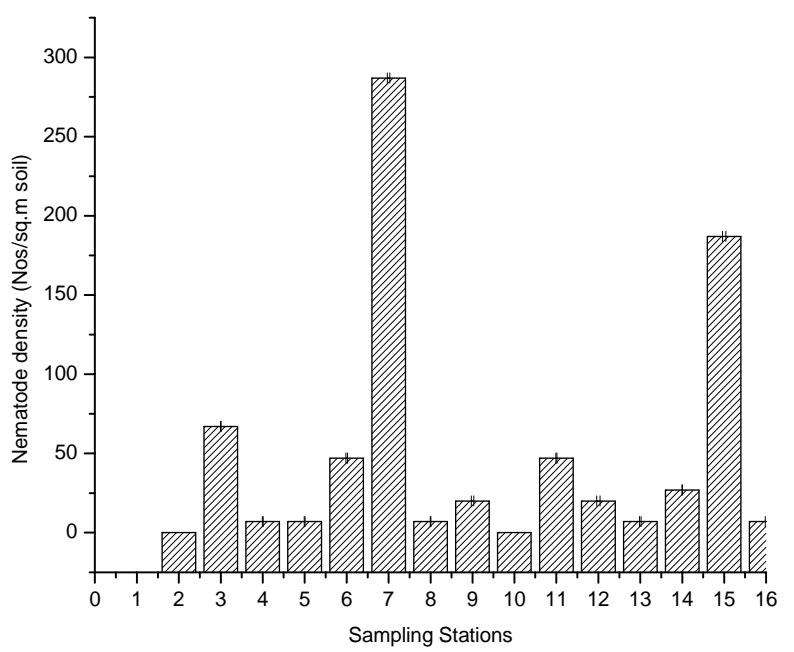

(a)

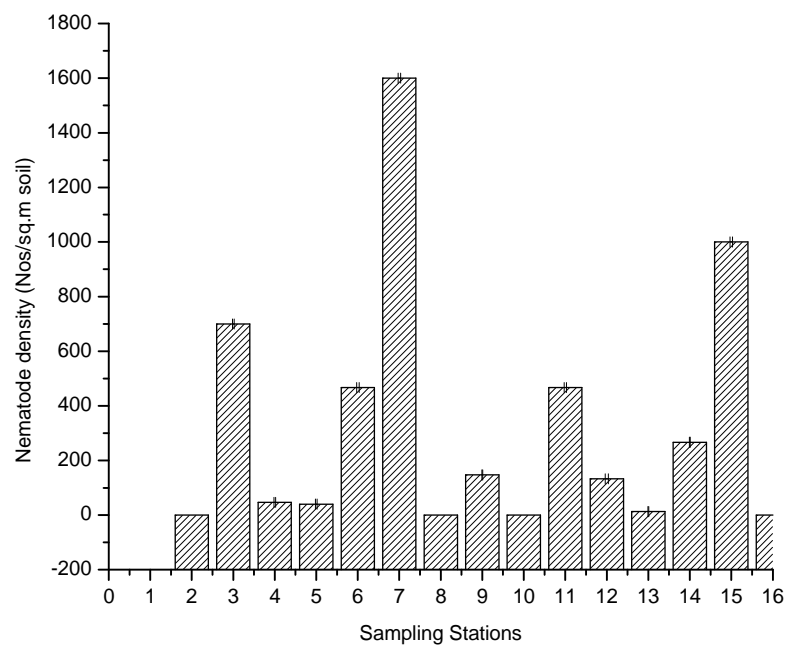

(b)

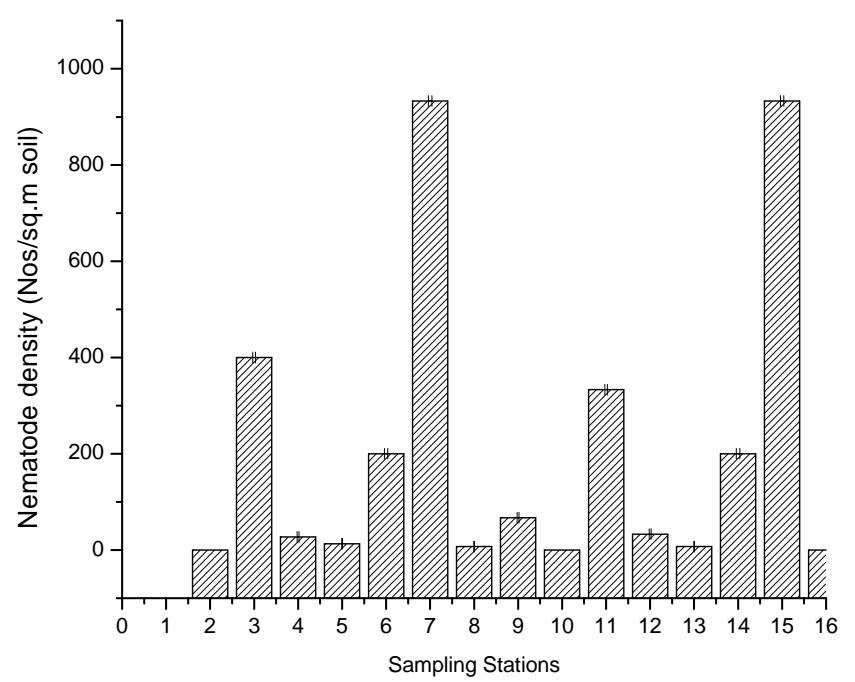

(c)

Figure 2. Nematode abundance in the Soils of Study stations during different seasons. (a) Pre-monsoon season; (b) Monsoon season; (c) Post-monsoon season.

the study area showed significant and positive correlation with nematode density. Smiles (1988) [10] in his studies concluded that the rate of soil water movement controls many biological activities such as wilting and germination of plants and hatching of nematode cysts and spores. The spatial variability of water holding capacity and nematode density during the pre-monsoon, monsoon and post monsoon seasons were plotted and the results are shown in Figure 3. Water holding capacity showed very high positive correlation with nematode density during the pre monsoon season in all the stations. But in the monsoon season due to the dilution factor, this positive correlation was very high in most of the study stations except in the stations S1, S2, S3, S4 and S16 where this positive correlation was only high. In the post-monsoon season, this positive correlation showed the same pattern as that in the monsoon season except for the stations- $\mathrm{S} 3$ and S4 where the positive correlation was reverted back to very high.

Moisture content in the soils of study stations showed a significant and positive correlation with nematode density. Townshend and Webber (1971) [11] showed that the movement and survival of nematodes was maximum in soils with maximum moisture retention. The spatial variability of moisture content and nematode density during the pre-monsoon, monsoon and post-monsoon seasons were plotted and the results are shown in Figure 4. Moisture content showed high positive correlation with nematode density during the pre monsoon season in most of the study stations except in the stations, S11, S12, S13, S14 and S15 where this positive correlation was very high. But in the monsoon season due to the dilution factor, this positive correlation was very high all the stations. In the post monsoon season, this positive correla- 
Table 5. (a) Correlation of nematode density with physico-chemical characteristics in rural area; (b) Correlation of nematode density with physico-chemical in urban area.

(a)

\begin{tabular}{|c|c|c|c|}
\hline Parameters & Pre monsoon & Monsoon & Post monsoon \\
\hline $\mathrm{pH}$ & $-0.680\left(^{* *}\right)$ & $-0.841\left(^{* *}\right)$ & $-0.820\left(^{* *}\right)$ \\
\hline Conductivity & $0.974\left(^{* *}\right)$ & $0.967\left(^{* *}\right)$ & $0.992\left(^{* *}\right)$ \\
\hline Temperature & -0.223 & -0.202 & 0.000 \\
\hline Pore space & $-0.612\left(^{* *}\right)$ & $-0.692\left(^{* *}\right)$ & $-0.662\left(^{* *}\right)$ \\
\hline Water holding capacity & $0.845\left(^{* *}\right)$ & $0.972\left(^{* *}\right)$ & $0.965\left(^{* *}\right)$ \\
\hline Bulk density & $-0.817\left(^{* *}\right)$ & $-0.933\left(^{* *}\right)$ & $-0.923\left(^{* *}\right)$ \\
\hline Moisture content & $0.798\left(^{* *}\right)$ & $0.892\left(^{* *}\right)$ & $0.875\left(^{* *}\right)$ \\
\hline Chloride & -0.245 & -0.288 & -0.277 \\
\hline Organic matter & $0.958\left(^{* *}\right)$ & $0.967\left(^{* *}\right)$ & $0.804\left(^{* *}\right)$ \\
\hline Total nitrogen & $0.719\left(^{* *}\right)$ & $0.980\left(^{* *}\right)$ & $0.821\left(^{* *}\right)$ \\
\hline Phosphorus & $0.990\left(^{* *}\right)$ & $0.907\left(^{* *}\right)$ & $0.766\left(^{* *}\right)$ \\
\hline Potassium & $0.680\left({ }^{* *}\right)$ & $0.567\left(^{* *}\right)$ & $0.497\left(^{* *}\right)$ \\
\hline Lead & $-0.563\left(^{* *}\right)$ & $-0.436(*)$ & $-0.774\left(^{* *}\right)$ \\
\hline Zinc & -0.311 & -0.183 & -0.254 \\
\hline Manganese & -0.275 & -0.020 & -0.237 \\
\hline Copper & 0.333 & $0.450\left(^{* *}\right)$ & $0.458\left(^{* *}\right)$ \\
\hline Chromium & $-0.464\left(^{* *}\right)$ & $-0.405\left(^{*}\right)$ & $-0.434\left(^{* *}\right)$ \\
\hline
\end{tabular}

(b)

\begin{tabular}{|c|c|c|c|}
\hline Parameters & Pre monsoon & Monsoon & Post monsoon \\
\hline $\mathrm{pH}$ & -0.307 & $-0.796\left(^{* *}\right)$ & $-0.819\left(^{* *}\right)$ \\
\hline Conductivity & $0.856\left(^{* *}\right)$ & $0.989\left(^{* *}\right)$ & $0.990\left(^{* *}\right)$ \\
\hline Temperature & $-0.616\left(^{* *}\right)$ & -0.016 & -0.059 \\
\hline Pore space & $-0.551\left(^{* *}\right)$ & $-0.641\left(^{* *}\right)$ & $-0.558\left(^{* *}\right)$ \\
\hline Water holding capacity & $0.915\left(^{* *}\right)$ & $0.978\left(^{* *}\right)$ & $0.961\left(^{* *}\right)$ \\
\hline Bulk density & $-0.893\left(^{* *}\right)$ & $-0.902\left(^{* *}\right)$ & $-0.886\left(^{* *}\right)$ \\
\hline Moisture content & $0.741\left(^{* *}\right)$ & $0.875\left(^{* *}\right)$ & $0.806\left(^{* *}\right)$ \\
\hline Chloride & -0.266 & -0.305 & -0.256 \\
\hline Organic matter & $0.828\left(^{* *}\right)$ & $0.860\left(^{* *}\right)$ & $0.756\left(^{* *}\right)$ \\
\hline Total nitrogen & $0.711\left(^{* *}\right)$ & $0.991\left(^{* *}\right)$ & $0.700\left(^{* *}\right)$ \\
\hline Phosphorus & $0.988\left(^{* *}\right)$ & $0.903\left(^{* *}\right)$ & $0.814\left(^{* *}\right)$ \\
\hline Potassium & $0.693\left(^{* *}\right)$ & $0.929\left(^{* *}\right)$ & $0.948\left(^{* *}\right)$ \\
\hline Lead & $-0.570\left(^{* *}\right)$ & $-0.528\left(^{* *}\right)$ & $-0.767\left(^{* *}\right)$ \\
\hline Zinc & $-0.354\left(^{*}\right)$ & -0.254 & -0.262 \\
\hline Manganese & $-0.313\left(^{*}\right)$ & -0.274 & $-0.537\left(^{* *}\right)$ \\
\hline Copper & $0.342\left(^{*}\right)$ & $0.438\left(^{* *}\right)$ & $0.377\left(^{*}\right)$ \\
\hline Chromium & -0.138 & -0.261 & $-0.455\left(^{* *}\right)$ \\
\hline
\end{tabular}

Table 6. Significance level of correlation coefficient.

\begin{tabular}{cc}
\hline Range & Significance \\
\hline $0.00-0.200$ & Negligible \\
$0.200-0.400$ & Low \\
$0.400-0.600$ & Moderate \\
$0.600-0.800$ & Substantial \\
$0.800-1.00$ & Very high \\
\hline
\end{tabular}

tion showed the same pattern as that in the monsoon season except for the stations, S7 and S8 where the positive correlation was high.

Organic matter content in the soils of study stations showed a significant and positive correlation with nematode density. This is in agreement with the studies by Gorres et al. (1998) [12] which showed that the soils rich in organic matter were reported with the highest nematode density. 


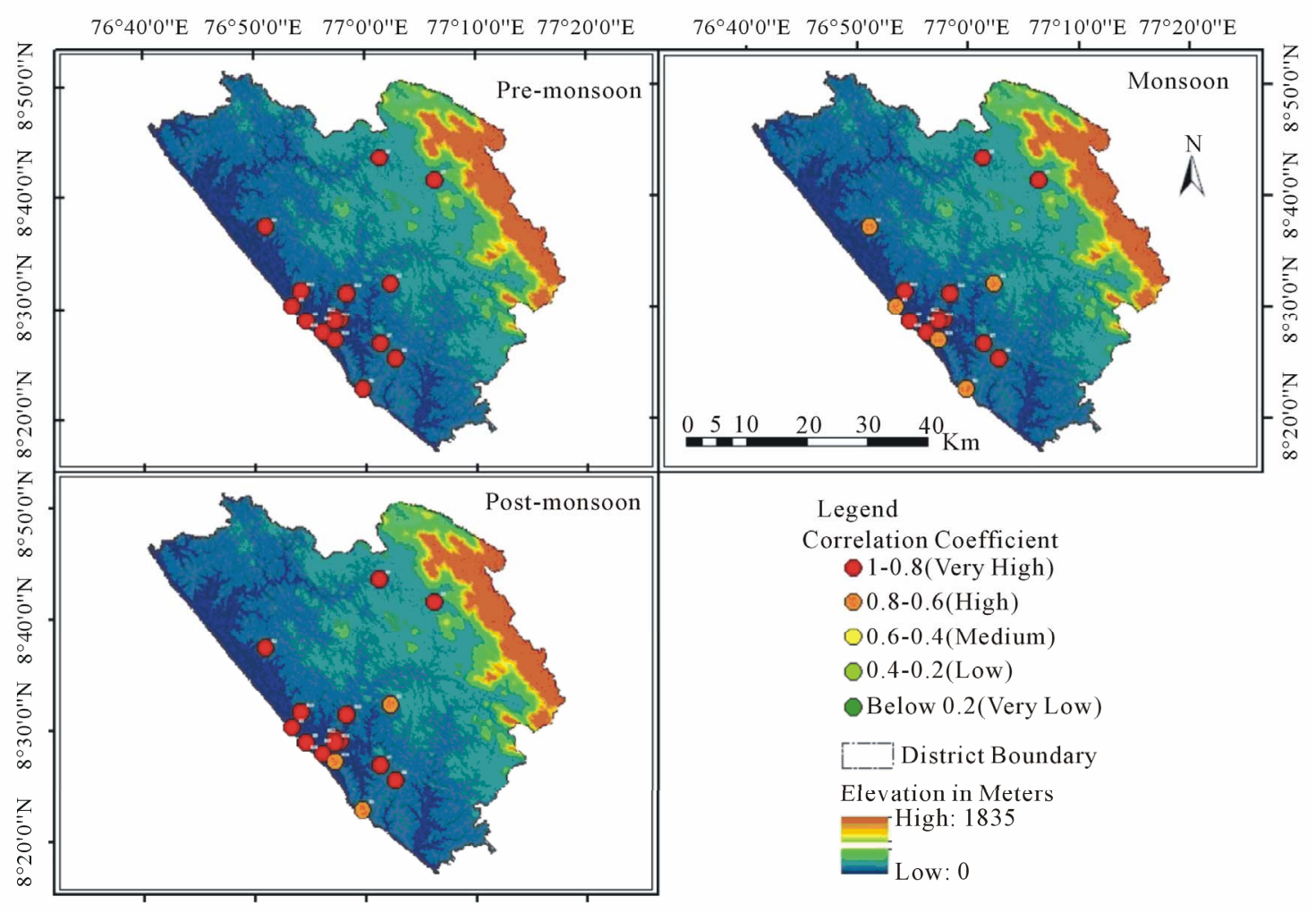

$76^{\circ} 40^{\prime} 0^{\prime \prime} \mathrm{E} \quad 76^{\circ} 50^{\prime} 0^{\prime \prime} \mathrm{E} \quad 77^{\circ} 0^{\prime} 0^{\prime \prime} \mathrm{E} \quad 77^{\circ} 10^{\prime} 0^{\prime \prime} \mathrm{E} 77^{\circ} 20^{\prime} 0^{\prime \prime} \mathrm{E}$

Figure 3. Mapping of water holding capacity and nematode density.

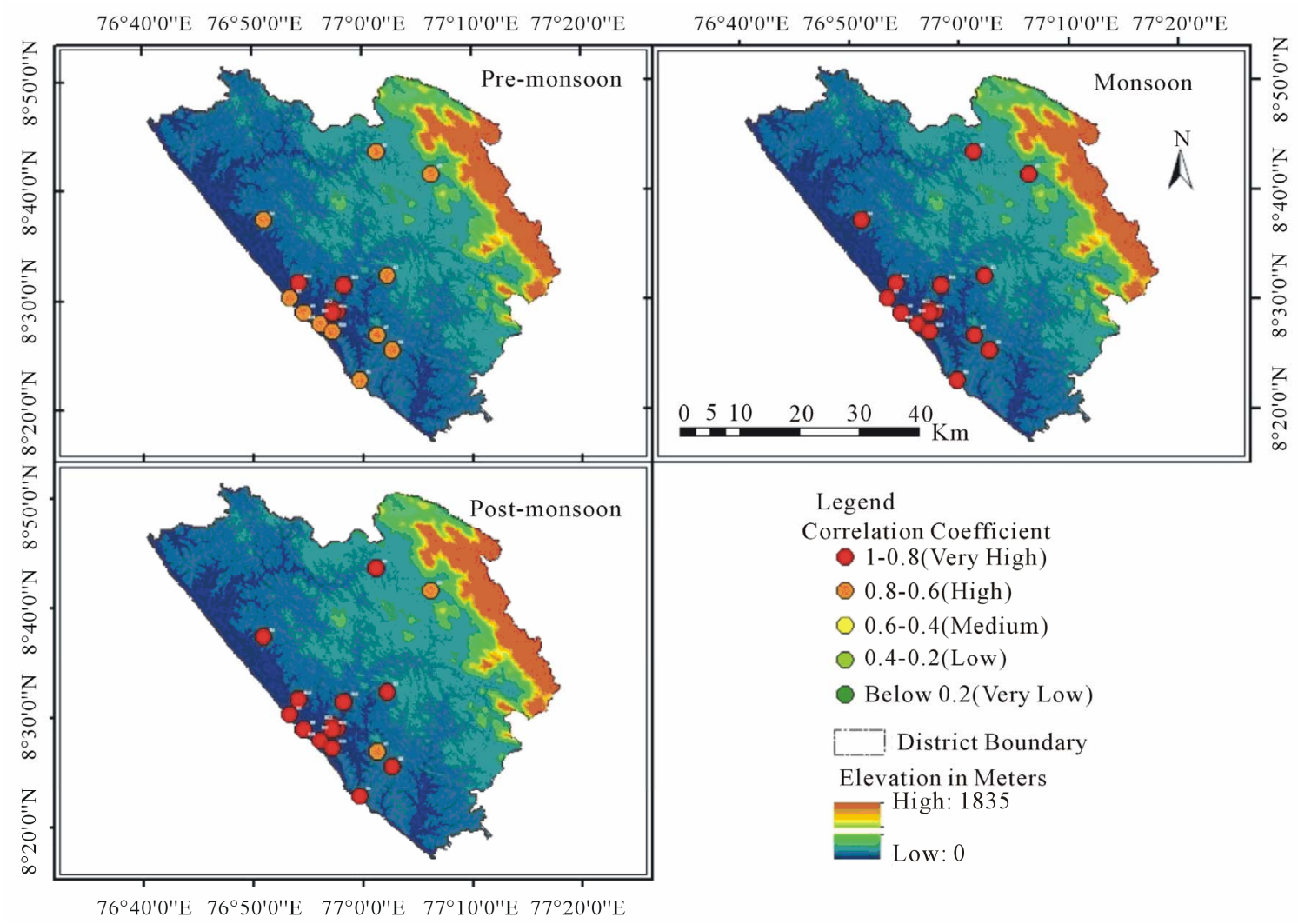

Figure 4. Mapping of moisture content and nematode density. 
The spatial variability of organic matter content and nematode density during the pre monsoon, monsoon and post monsoon seasons were plotted and the results are shown in Figure 5. Organic matter content showed very high positive correlation with nematode density during the pre monsoon and monsoon seasons in all the stations. In the post monsoon season, this positive correlation showed the same pattern as that in the pre monsoon and monsoon seasons except for the stations, S7 and S9 where the positive correlation was high.

\subsection{Mapping of Significantly Negatively Correlated Physico-Chemical Characteristics and Nematode Density in Soil}

There is a significant and negative correlation between soil $\mathrm{pH}$ and nematode density. This is in agreement with the studies conducted by Murialdo et al. (2002) [13] which showed that when the soil $\mathrm{pH}$ was high, nematode density was low. The spatial variability of $\mathrm{pH}$ content in soil and nematode density during the pre monsoon, monsoon and post monsoon seasons were plotted and the results are shown in Figure 6. Soil pH showed very high negative correlation with nematode density during the pre monsoon season in stations, S13, S11, S12, S14 and S15. But in the monsoon season due to the dilution factor, this negative correlation was reduced. In the post monsoon season, the correlation showed the same pattern as that in the monsoon season.

Zinc content in the soils of study stations showed a significant and negative correlation with nematode density. Studies by Shao et al. (2008) [14] showed that nematode densities were negatively correlated with zinc concentrations as their population densities decreased with increased zinc concentrations. The spatial variability of zinc content and nematode density during the pre monsoon, monsoon and post monsoon seasons were plotted and the results are shown in Figure 7. Zinc content showed high negative correlation with nematode density during the pre monsoon season in the stations, S10, S11, S12 and S13. Due to the dilution factor in the monsoon season, this negative correlation was reduced in all the stations. But this negative correlation was very high in the stations, S14 and S15 in the monsoon season. The effect of raised water holding capacity and moisture content in the monsoon season influenced the soils in the station, S14 resulting in very high negative correlation. In the station, S15 the retention of zinc content in soil will be more due to the dilution factor resulting in the high negative correlation with nematode density. But in the post monsoon season due to the less water content in these soils, the negative correlation will be lesser.

Manganese content in the soils of study stations showed a significant and negative correlation with nematode den- sity. Studies by Norton and Hoffmann (1974) [15] had shown that a negative correlation was observed between manganese content and egg/cyst ratio of nematodes which in turn will be reflected in their population densities. The spatial variability of manganese content in the soils and nematode density during the pre monsoon, monsoon and post monsoon seasons were plotted and the results are shown in Figure 8. Manganese content in soils showed the same negative correlation pattern with nematode density in both the pre monsoon and monsoon seasons. But in the post monsoon season, this negative correlation was very high in the station, S14 as these clayey soils were with less water content at this season as compared to that in the monsoon season and will augment the manganese content in soils. This is in agreement with the studies conducted by Rajmohan and Elango (2005) [16] which concluded that soils with high clay content will retain more manganese especially in the post monsoon season. In stations, S4, S6 and S9, the negative correlation was high. In station, S6 with high clay content in soils, the manganese retention will be more in this season. As S6 is a rural market area and S14 is an urban market area, the manganese contamination will be more in the urban market area due to different anthropogenic activities compared to the corresponding rural area. So station, S6 showed only high level of correlation with nematode density in the post monsoon season. In station, S4 which is a road-side area, the manganese deposition will be more in the post monsoon season. Previous studies by Singh and Singh (2006) [17] had shown that the manganese deposition in soils will be more in the post monsoon season in the road-side soils in India. Station, S9 which is an urban coastal area, this negative correlation will be more due to the hyper saline nature of these soils. This is in agreement with the studies by Alongi et al. (2005) [18] which showed that hyper saline soils will retain more manganese content in soils during the post monsoon season.

\section{Conclusion}

The soil physico-chemical characteristics which showed significant (positive and negative) correlations with nematode density were spatially plotted using the Arc GIS 10 to show their spatial variability. Water holding capacity, moisture content and organic matter content showed a significant and positive correlation with nematode density. PH, zinc content and manganese content showed a significant and negative correlation with nematode density. These soil mapping plots showed variations in the monsoon season compared to the pre monsoon and post monsoon seasons due to the dilution factor. In the study area, the organic matter content of urban soils with industrial activities and gasoline service stations was found to be significantly less compared to that of the rural area 


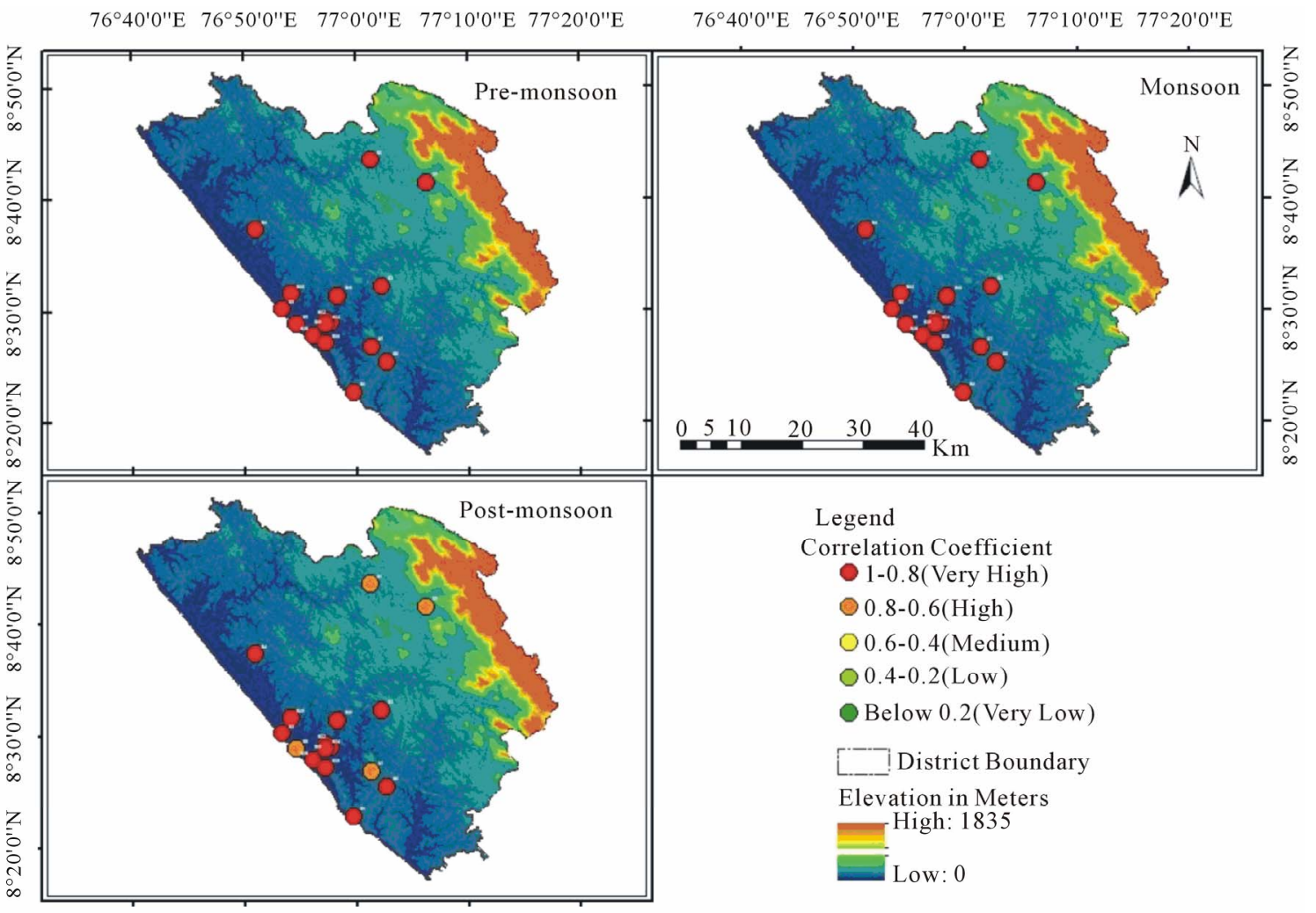

Figure 5. Mapping of organic matter content and nematode density.

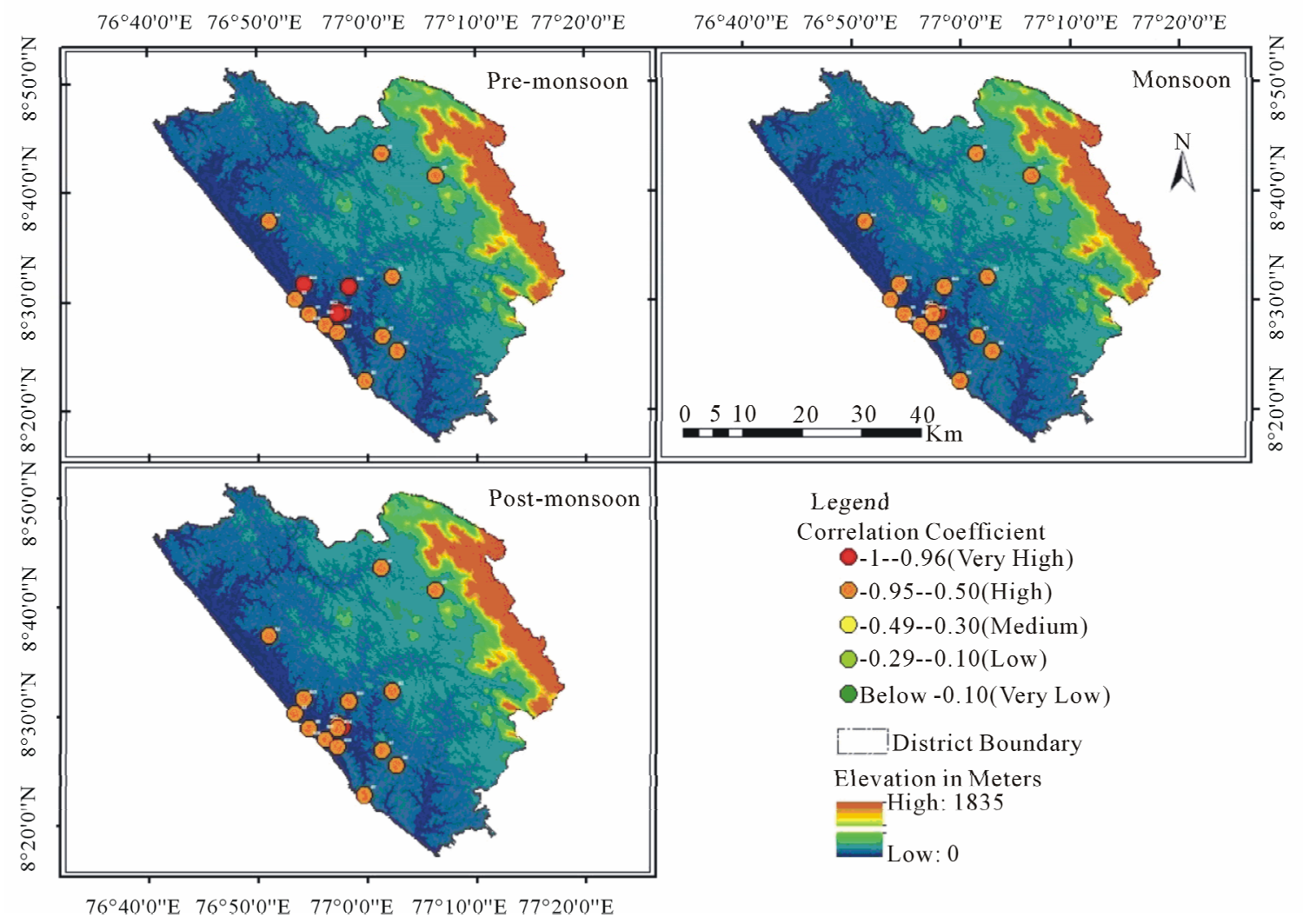

Figure 6. Mapping of $\mathrm{pH}$ and nematode density. 


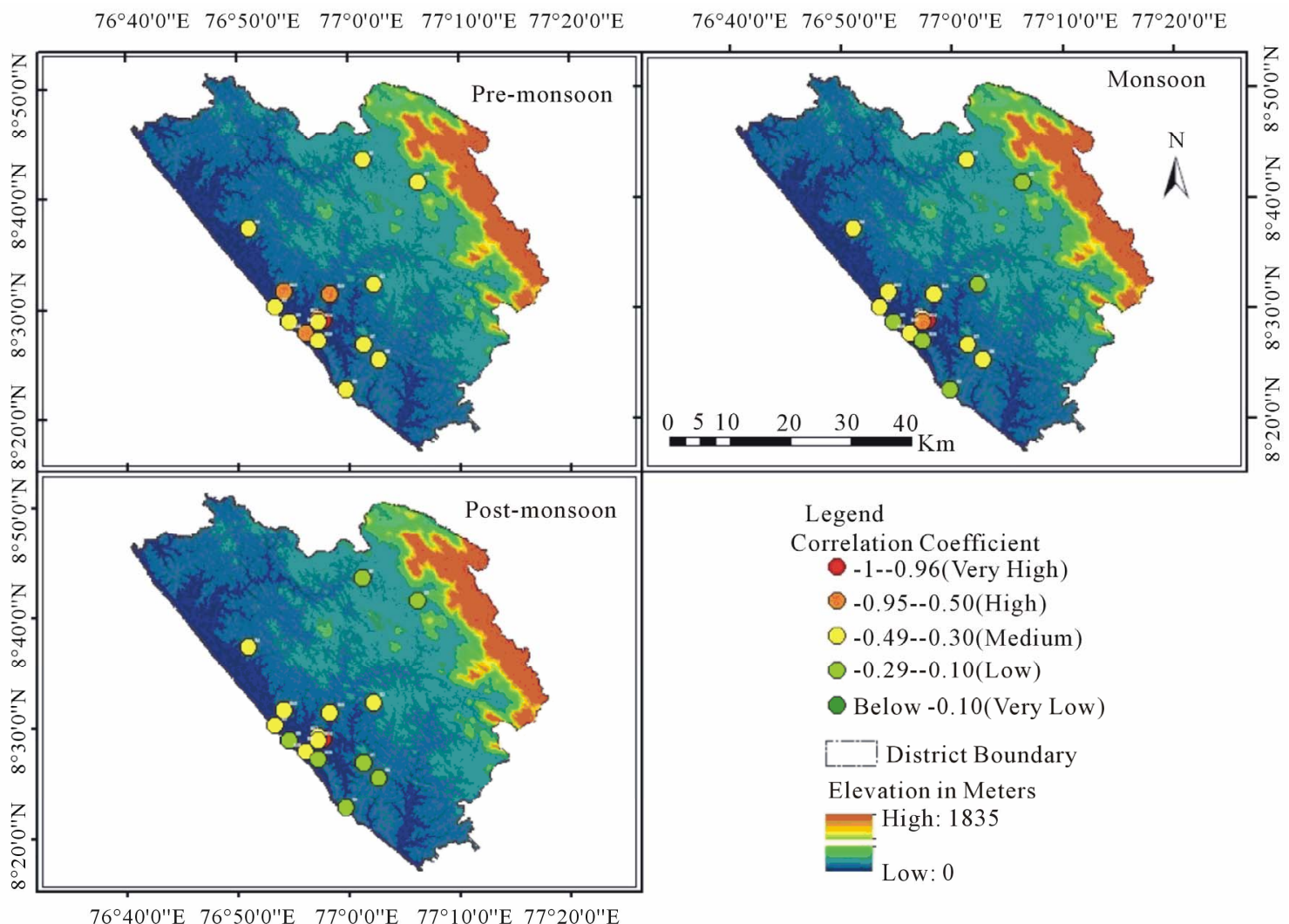

Figure 7. Mapping of zinc content and nematode density.

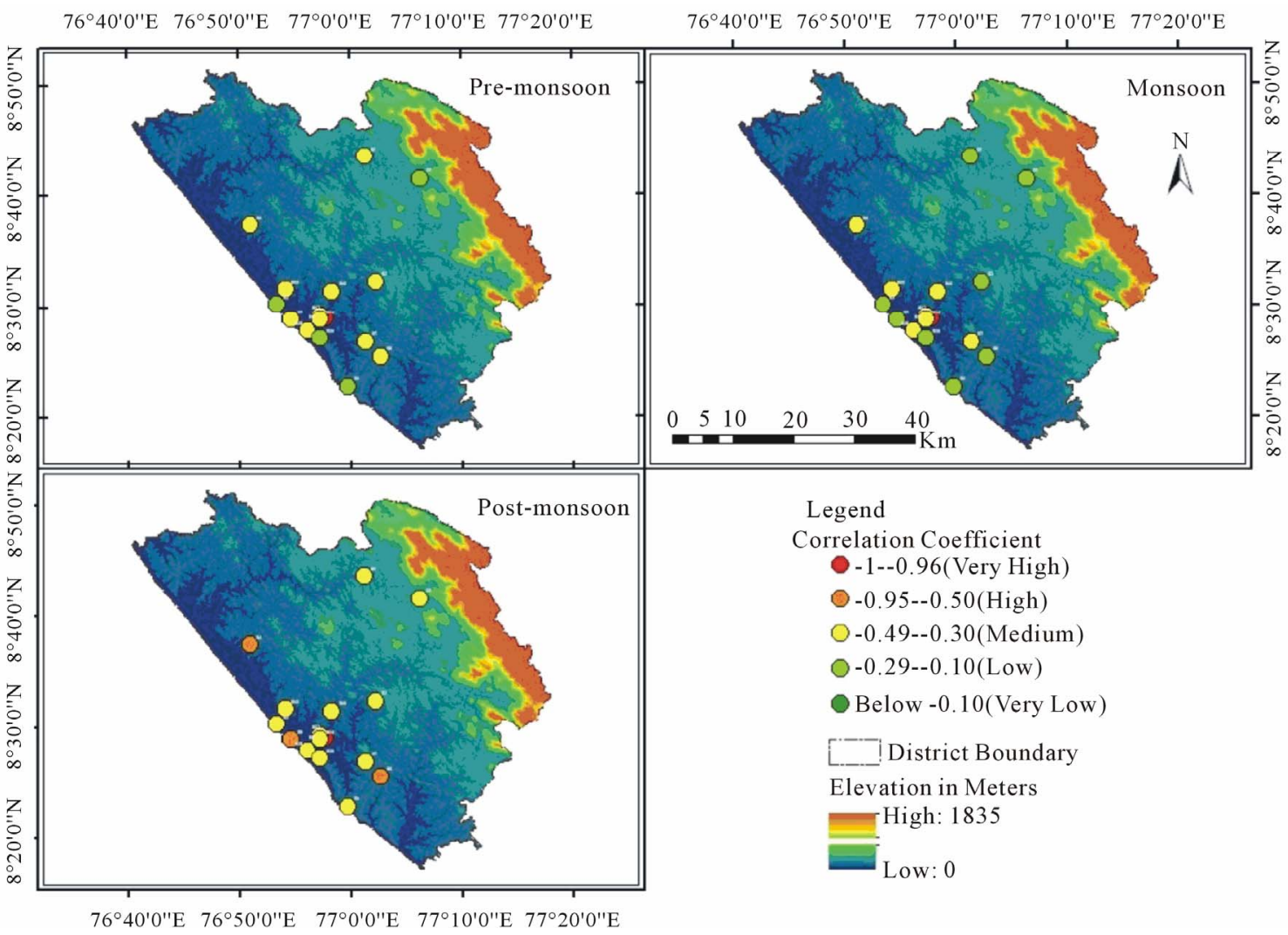

Figure 8. Mapping of manganese content and nematode density. 
soils with the same land use patterns. Also the urban area soils were prone to more heavy metal contamination than the rural area soils especially in the gasoline station and roadside areas of Thiruvananthapuram District. This will reduce the soil microbial activity and soil health which in turn will be reflected in the crop production. This soil quality mapping provides a basis for identifying such tension zones and serves as a triggering mechanism for implementation of mitigating strategies.

\section{Acknowledgements}

The authors sincerely thank Prof. M.S. Rajasree, Director, Indian Institute of Information Technology, TechnoPark, Thiruvananthapuram for providing facilities to conduct the spatial studies. Also extend sincere thanks to Mr. Vishnu, Project Fellow, Indian Institute of Information Technology, Thiruvananthapuram for the help rendered in doing the spatial analysis.

\section{REFERENCES}

[1] B. J. Alloway, "Pollution-Causes, Effects and Control," 3rd Edition, The Royal Society of Chemistry Publishers, Cambridge, 1996.

[2] J. V. Tarazona, M. D. Fernández and M. M. Vega, "Regulation of Contaminated Soils in Spain e a New Legal Instrument," Journal of Soils Sediments, Vol. 5, No. 3, 2005, pp. 121-124.

[3] M. J. Fortin and M. Dale, "Spatial Analysis-A Guide for Ecologists," The Edinburgh Building, Published by Cambridge University, Cambridge, 2005.

[4] P. K. Gupta, "Soil, Plant, Water and Fertiliser Analysis," Agro Botanica Publishers and Distributors, New Delhi, 1999, p. 438.

[5] N. N. Saxena, "Environmental Analysis of Water, Soil and Air," Agro Botanical Publishers, 1994.

[6] G. B. Korte, "The GIS Book," 5th Edition, SAN 6940269, On Word Press Publishers, 2001.

[7] N. A. Cobb, "Notes on Nematology," Controlled Science Nematology, Vol. 5, No. 5, 1917, pp. 117-128.

[8] T. R. E. Southwood and P. A. Henderson Ecological Methods," Published by Blackwell Science Ltd., London, 2000, pp. 218-240.
[9] J. B. Goodey, "Laboratory Methods for Work with Plant and Soil Nematodes, Technical Bulletin No.2, Ministry of Agriculture, Fisheries and Food," Nematology Department, Rothamsted Experimental Station, Harpenden, Herts, London, 1963, pp. 21-22.

[10] D. E. Smiles, "Aspects of the Physical Environment of Soil Organisms," Biology and Fertility of Soils, Vol. 6, No. 3, 1988, pp. 204-215.

[11] J. L. Townshend and L. R. Webber, "Movement of Pratylenchuspenetrans and the Moisture Characteristics of Three Ontario Soils," Nematologica, Vol. 17, No. 1, 1971, pp. 47-57. http://dx.doi.org/10.1163/187529271X00404

[12] J. F. Gorres, M. J. Dichiaro, J. B. Lyons and J. A. Amador, "Spatial and Temporal Patterns of Soil Biological Activity in a Forest and an Old Field," Soil Biological Biochemistry, Vol. 30, No. 2, 1998, pp. 219-230.

[13] S. E. Murialdo, M. G. Fuertes, J. F. Gonza'lez and E. Chavez, "Nematodes as Indicators of Wetland Pollution," Journal of Environmental Biology, Vol. 23, No. 4, 2002, pp. 423-428.

[14] Y. Shao, W. Zhang, J. Shen, L. Zhou, H. Xia, W. Shu, H. Ferris and S. Fu, "Nematodes as Indicators of Soil Recovery in Tailings of a Lead/Zinc Mine," Soil Biological Biochemistry, Vol. 40, No. 2, 2008, pp. 2040-2046.

[15] D. C. Norton and J. K. Hoffmann, "Distribution of Selected Plant Parasitic Nematodes Relative to Vegetation and Edaphic Factors," Journal of Nematology, Vol. 6, No. 1, 1974, pp. 81-86.

[16] N. Rajmohan and L. Elango, "Distribution of Iron, Manganese, Zinc and Atrazine in Groundwater in Parts of Palar and Cheyyar River Basins, South India," Journal of Environmental and Monitoring Assessment, Vol. 107, No. 1-3, 2005, pp. 115-131. http://dx.doi.org/10.1007/s10661-005-5307-0

[17] A. K. Singh and M. Singh, "Lead Decline in the Indian Environment Resulting from the Petrol-Lead Phase-Out Programme," Science Total Environment, Vol. 368, No. 2-3, 2006, pp. 686-694. http://dx.doi.org/10.1016/j.scitotenv.2006.04.013

[18] D. M. Alongi, A. L. Ramanathan, L. Kannan, F. Tirendi, L. A. Trott and M. Bala Krishna Prasad, "Influence of Human-Induced Disturbance on Benthic Microbial Metabolism in the Pichavaram Mangroves, Vellar-Coleroon Estuarine Complex, India," Marine Biology, Vol. 147, No. 4, 2005, pp. 1033-1044. http://dx.doi.org/10.1007/s00227-005-1634-5 